\title{
Dynamics and Bifurcation of a Model for Hormonal Control of the Menstrual Cycle with Inhibin Delay*
}

\author{
Alison Margolskee \\ Department of Mathematics \\ North Carolina State University \\ e-mail: amargol@ncsu.edu \\ and \\ James F. Selgrade \\ Department of Mathematics and Biomathematics Program \\ North Carolina State University, Raleigh, NC 27695-8205 \\ e-mail: selgrade@math.ncsu.edu
}

\begin{abstract}
A system of 13 ordinary differential equations with 42 parameters is presented to model hormonal regulation of the menstrual cycle. The model's fit to data is improved by including a time delay for the effect of inhibin on the synthesis of follicle stimulating hormone. Biological reasons for this improvement are discussed. Bifurcations with respect to changes in three important parameters are examined. One parameter represents the level of estradiol adequate for significant synthesis of luteinizing hormone. Bifurcation diagrams with respect to this parameter reveal an interval of parameter values for which a unique stable periodic solution exists and this solution represents a menstrual cycle during which ovulation occurs. The second parameter measures mass transfer between the first two stages of ovarian development and is indicative of healthy follicular growth. The third parameter is the time delay. Changes in the second parameter and the time delay affect the size of the uniqueness interval defined with respect to the first parameter. Saddle-node, transcritical and degenerate Hopf bifurcations are studied.
\end{abstract}

Key Words. Estradiol, follicle, Hill function, degenerate Hopf bifurcation

AMS 2000 Mathematics Subject Classification: 92D25, 39A11

\footnotetext{
* Research supported by NSF grant DMS-0920927.
} 


\section{Introduction.}

Systems of ordinary and delayed differential equations have been used to model hormonal regulation of the human menstrual cycle, e.g., see Bogumil et al., 1972a, 1972b [3, 4], McIntosh and McIntosh, 1980 [15], Plouffe and Luxenberg, 1992 [20], Selgrade and Schlosser, 1999 [25], Schlosser and Selgrade, 2000 [22], Harris-Clark et al., 2003 [10], Reinecke and Deuflhard, 2007 [21], and Pasteur, 2008 [19]. Dual control of the menstrual cycle depends on hormones produced by the hypothalamus and the pituitary glands in the brain and by the ovaries. The pituitary secretes follicle stimulating hormone $(F S H)$ and luteinizing hormone $(L H)$ which control ovarian development and ovulation, see [11, 29, 30]. The ovaries produce estradiol $\left(E_{2}\right)$, progesterone $\left(P_{4}\right)$ and inhibin (Inh) which affect the synthesis and release of $F S H$ and $L H$, see [12, 14, 26]. Harris, Pasteur, Schlosser and Selgrade [10, 19, 22, 25] have derived a 13-dimensional system of delayed differential equations which captures these interacting mechanisms. Model parameters were identified using two different clinical data sets for normally cycling women (McLachlan et al., 1990 [16], and Welt et al., 1999 [27]). Model simulations with parameters from the McLachlan data [16] revealed two stable periodic solutions [10] — one fitting the McLachlan data for normally cycling women and the other being non-ovulatory because of no $L H$ surge. The non-ovulatory cycle has similarities to an abnormal cycle of a woman with polycystic ovarian syndrome (PCOS) 28, the leading cause of female infertility. However, model simulations corresponding to the Welt parameters produced only one stable periodic solution and it fits the Welt data for normally cycling women. Selgrade et al., 2009 24], explained this apparent inconsistency by showing that a change in only one sensitive parameter of the Welt system would result in the Welt model exhibiting bistability like the McLachlan model.

Abnormal cycling and non-ovulatory cycling have serious health and reproductive consequences. In fact, between $6 \%$ and $9 \%$ of adult women exhibit some symptoms of PCOS, see Azziz et al., 2004 [2], and Alvarez-Blasco et al., 2006 [1]. Since cycle irregularities are usually associated with abnormal hormone levels, mathematical models of hormonal regulation may provide information about parameter variations which result in abnormal cycling and may provide insights about possible hormonal therapies. In an effort to understand what parameter ranges result in normal and abnormal cycling, Selgrade [23] set the time-delays to zero in the Welt model and used the software XPPAUT [7] to study bifurcation diagrams with respect to two of the most sensitive parameters. Bifurcation diagrams for the resulting autonomous system could be drawn with the features of AUTO [5] in XPPAUT. This autonomous model gives an acceptable fit to the 28 day Welt data set [27] except some hormone peaks are lower than the data and the period for the normal cycle is only about 26 days, see Figure 1. 

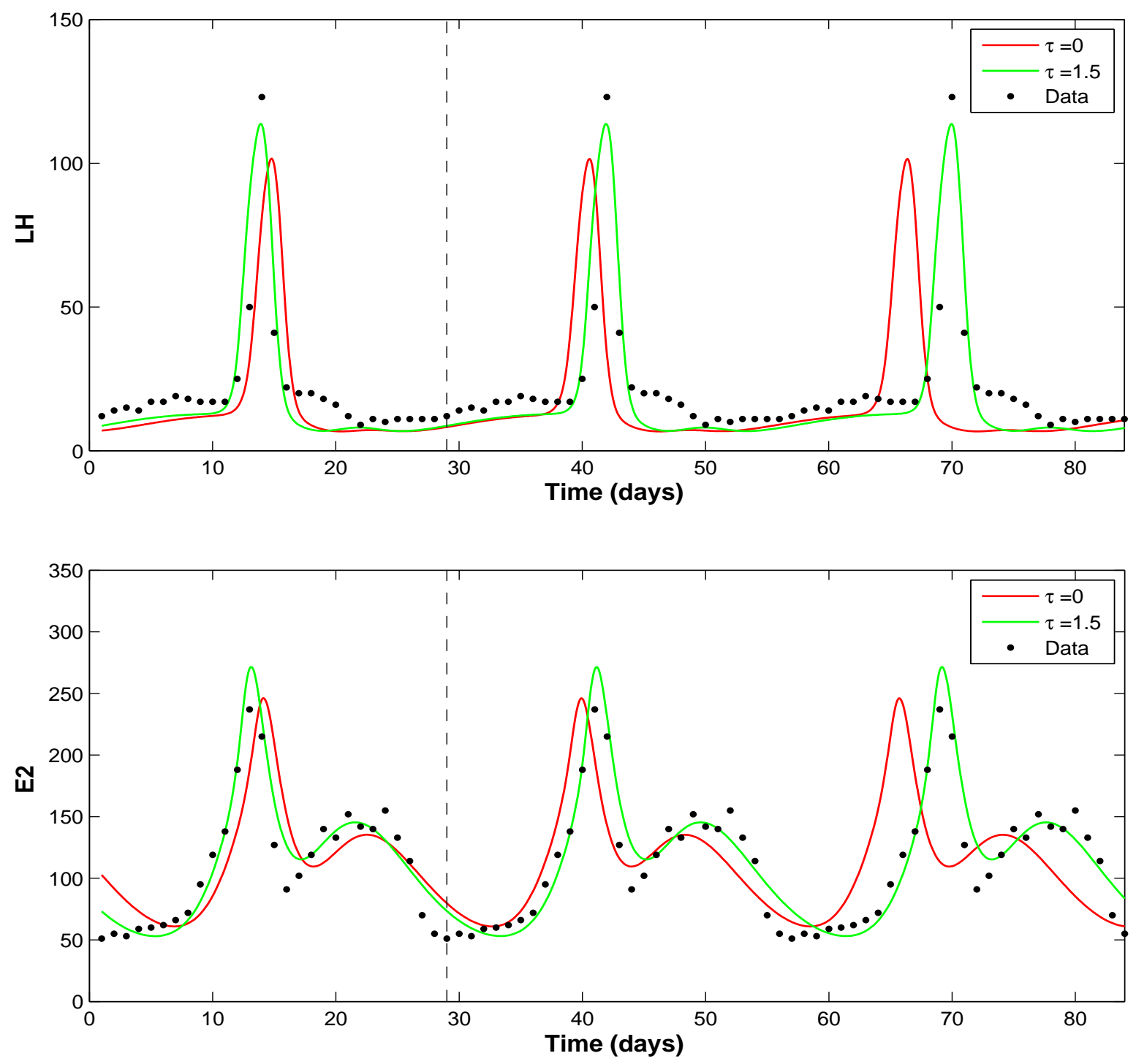

Figure 1: $\quad L H$ and $E_{2}$ simulations for 3 cycles of the Welt model with inhibin delay $\tau$ of 1.5 days (green curves) and no delay (red curves) with data points ( 84 black dots) corresponding to the 28 day data from Welt et al. [27] plotted 3 times. The vertical dashed line indicates day 29, where the second cycle begins and where both solution orbits are very close to one another. 
The two key parameters for analysis in [23] are $K m_{L H}$ and $c_{2}$. $K m_{L H}$ represents the level of $E_{2}$ sufficient for significant $L H$ synthesis and the $L H$ surge and $c_{2}$ indicates the ovarian mass transfer rate between the first two stages of ovarian development. The bifurcation diagram with respect to $K m_{L H}$ reveals an interval of $K m_{L H}$ parameter values for which a unique stable periodic solution exists and this solution represents a menstrual cycle with a $L H$ surge adequate for ovulation. If $K m_{L H}$ lies outside this cycle uniqueness interval then either no $L H$ surge occurs or there are two stable cycles - one is ovulatory and the other is non-ovulatory. Changes in $c_{2}$ affect the size of this interval because of the positions of Hopf, saddle-node and transcritical bifurcations as discussed in [23].

In this study, we carry out a bifurcation analysis for the system of delayed differential equations using the DDEBIFTOOL [6], which is designed to handle the delay. The original model [10] had three discrete time-delays (one corresponding to each ovarian hormone) which represented the time interval between changes in ovarian hormone concentrations and subsequent changes they cause in synthesis rates of the pituitary hormones. Here we show that including only a delay of $\tau=1.5$ days for inhibin is needed to improve the fit to the Welt data, see Figure 1. The other two delays were no more than a day and did not contribute significant additional improvement. Also, the system with the inhibin delay has larger uniqueness intervals than the model with no delay (see Table 2). In Section 3 we speculate about the biological reasons for this improvement in model behavior due to inhibin delay. We examine bifurcation diagrams with respect to $K m_{L H}$ for the delayed system and show that the cycle uniqueness interval is usually determined by two saddlenode bifurcations. For the delay $\tau$ fixed at 1.5 days, we illustrate how this interval may be enlarged by varying $c_{2}$ due to the occurrence of two degenerate Hopf bifurcations. Then for fixed $c_{2}$, we increase the delay parameter $\tau$ from 0 to 1.5 to unfold transcritical bifurcations and produce large cycle uniqueness intervals. Finally, we illustrate how loops in the $K m_{L H}$ bifurcation diagrams may appear and disappear by varying the parameters $\tau$ and $c_{2}$.

\section{Biological Background and Model Equations.}

The menstrual cycle for an adult female consists of the follicular phase, ovulation, the luteal phase and menstruation (e.g., see Odell [17] or Ojeda [18]). During the follicular phase, FSH produced by the pituitary gland promotes the development of 6 to 12 follicles. Typically one dominant follicle is selected to grow to maturity and to produce a large amount of $E_{2}$ which primes the pituitary for $L H$ secretion. At mid-cycle, a surge of $L H$ over a period of 4 or 5 days results in ovulation. After releasing its egg, the dominant follicle becomes the corpus luteum which produces hormones in preparation for pregnancy and produces $P_{4}$ and $I n h$ which inhibit $L H$ and $F S H$, respectively. If fertilization does not occur, the corpus luteum atrophies, menstruation follows and a rise in FSH marks 
the beginning of the next cycle.

Harris, Pasteur, Schlosser and Selgrade [9, 10, 19, 22, 25] developed a model for this endocrine control system based on 13 ordinary differential equations (S) with three auxiliary equations (A) and with discrete time-delays. Four of these differential equations (S1-S4) describe the synthesis, release and clearance of $L H$ and $F S H$. The state variables $R P_{L H}$ and $R P_{F S H}$ represent the amounts of these hormones in the pituitary and $L H$ and $F S H$ represent the blood concentrations of these hormones. The biological literature (e.g., [12, 14, 29]) indicates that $L H$ exhibits a biphasic response to $E_{2}$. To capture this our model assumes that the effect of $E_{2}$ on $L H$ synthesis is different than the effect on $L H$ release, i.e., $E_{2}$ inhibits release (see the denominator of the second term in (S1)) but at high levels $E_{2}$ promotes synthesis (see the Hill function in the numerator of the first term of (S1)). On the other hand, $P_{4}$ inhibits $L H$ synthesis but promotes release. The release term appears in (S1) as a decay term and in (S2) as a growth term, where it is divided by blood volume $v$. The equations (S3-S4) for FSH are similar except the synthesis term has Inh inhibition which is delayed by time $\tau$. The parameters in (S1-S4) are named according to the traditional usage for chemical reactions, e.g., $V_{1, L H}$ denotes the velocity of the reaction (see Keener and Sneyd, 2009, [13]).

The state variables in (S5-S13) represent tissue masses of 9 distinct stages of the ovary during the follicular and luteal phases of the cycle. ReF, SeF and $\operatorname{PrF}$ denote the recruited follicles, the secondary follicles and the preovulatory or dominant follicle, respectively. $O v_{1}$ and $O v_{2}$ represent periovulatory stages and $L u t_{i}, i=1, \ldots, 4$, denote four luteal stages. $L H$ and $F S H$ promote tissue growth within a stage and the transformation of tissue from one stage to the next. Since clearance from the blood of the ovarian hormones is on a fast time scale, we assume that blood levels of $E_{2}, P_{4}$, and $I n h$ are at quasi-steady state [13] as did Bogumil et al. [3]. Hence, we take these concentrations to be proportional to the tissue masses during the appropriate stages of the cycle giving the three auxiliary equations (A1-A3) for the ovarian hormones. Here we study the 13-dimensional system $(\mathrm{S})$ with $(\mathrm{A})$, which has one time-delay $\tau$. The forty-two parameters are listed in Table 1 and correspond to those which Selgrade [23] used to analyze bifurcation diagrams for the Welt system with time-delay set to zero.

\section{Auxiliary Equations (A)}

$$
\begin{aligned}
E_{2} & =e_{0}+e_{1} \text { SeF }+e_{2} \operatorname{PrF}+e_{3} \text { Lut }_{4} \\
P_{4} & =p_{0}+p_{1} \text { Lut }_{3}+p_{2} \text { Lut }_{4} \\
\text { Inh } & =h_{0}+h_{1} \operatorname{PrF}+h_{2} \text { Lut }_{2}+h_{3} \text { Lut }_{3} .
\end{aligned}
$$




\section{System (S)}

$$
\begin{aligned}
& \frac{d}{d t} R P_{L H}=\frac{V_{0, L H}+\frac{V_{1, L H} E_{2}^{8}}{K m_{L H}^{8}+E_{2}^{8}}}{1+P_{4} / K i_{L H, P}}-\frac{k_{L H}\left[1+c_{L H, P} P_{4}\right] R P_{L H}}{1+c_{L H, E} E_{2}} \\
& \frac{d}{d t} L H=\frac{1}{v} \frac{k_{L H}\left[1+c_{L H, P} P_{4}\right] R P_{L H}}{1+c_{L H, E} E_{2}}-a_{L H} L H \\
& \frac{d}{d t} R P_{F S H}=\frac{V_{F S H}}{1+\operatorname{Inh}(t-\tau) / K i_{F S H, I n h}}-\frac{k_{F S H}\left[1+c_{F S H, P} P_{4}\right] R P_{F S H}}{1+c_{F S H, E} E_{2}^{2}} \\
& \frac{d}{d t} F S H=\frac{1}{v} \frac{k_{F S H}\left[1+c_{F S H, P} P_{4}\right] R P_{F S H}}{1+c_{F S H, E} E_{2}^{2}}-a_{F S H} F S H \\
& \frac{d}{d t} R e F=b F S H+\left[c_{1} F S H-c_{2} L H^{\alpha}\right] R e F \\
& \frac{d}{d t} S e F=c_{2} L H^{\alpha} R e F+\left[c_{3} L H^{\beta}-c_{4} L H\right] S e F \\
& \frac{d}{d t} \operatorname{PrF}=c_{4} L H S e F-c_{5} L H^{\gamma} \operatorname{PrF} \\
& \frac{d}{d t} O v_{1}=c_{5} L H^{\gamma} \operatorname{PrF}-d_{1} O v_{1} \\
& \frac{d}{d t} O v_{2}=d_{1} O v_{1}-d_{2} O v_{2} \\
& \frac{d}{d t} L u t_{1}=d_{2} O v_{2}-k_{1} L u t_{1} \\
& \frac{d}{d t} L u t_{2}=k_{1} L u t_{1}-k_{2} L u t_{2} \\
& \frac{d}{d t} L u t_{3}=k_{2} L u t_{2}-k_{3} L u t_{3} \\
& \frac{d}{d t} L u t_{4}=k_{3} L u t_{3}-k_{4} L_{u} t_{4} \text {. }
\end{aligned}
$$


Table 1: Parameters and values for system (S) and auxiliary equations (A).

\begin{tabular}{|l|l|}
\hline \multicolumn{2}{|c|}{ Eqs. (S1-S4) } \\
\hline \hline$\tau$ & 1.5 days $^{-1}$ \\
\hline$k_{L H}$ & 2.42 day $^{-1}$ \\
\hline$a_{L H}$ & $14.0 \mathrm{day}^{-1}$ \\
\hline$V_{0, L H}$ & $500 \mathrm{IU} /$ day \\
\hline$V_{1, L H}$ & $4500 \mathrm{IU} /$ day \\
\hline$K m_{L H}$ & $200 \mathrm{pg} / \mathrm{mL}$ \\
\hline$K i_{L H, P}$ & $12.2 \mathrm{ng} / \mathrm{mL}$ \\
\hline$c_{L H, E}$ & $0.004 \mathrm{~mL} / \mathrm{pg}$ \\
\hline$c_{L H, P}$ & $0.26 \mathrm{~mL} / \mathrm{ng}$ \\
\hline$V_{F S H}$ & $375 \mathrm{IU} / \mathrm{day}$ \\
\hline$a_{F S H}$ & $8.21 \mathrm{day}-1$ \\
\hline$k_{F S H}$ & $1.90 \mathrm{day}{ }^{-1}$ \\
\hline$c_{F S H, E}$ & $0.0018 \mathrm{~mL} / \mathrm{pg}^{2}$ \\
\hline$K i_{F S H, \text { Inh }}$ & $3.5 \mathrm{IU} / \mathrm{mL}^{2}$ \\
\hline$c_{F S H, P}$ & $12.0 \mathrm{~mL} / \mathrm{ng}$ \\
\hline$v$ & $2.50 \mathrm{~L}$ \\
\hline
\end{tabular}

\begin{tabular}{|l|l|}
\hline \multicolumn{2}{|c|}{ Eqs. (S5-S13) } \\
\hline \hline$b$ & $0.05 \mathrm{~L} \mu \mathrm{g} /(\mathrm{IU}$ day $)$ \\
\hline$c_{1}$ & $0.08 \mathrm{~L} /(\mathrm{IU}$ day $)$ \\
\hline$c_{2}$ & $0.07(\mathrm{~L} / \mathrm{IU})^{\alpha} /$ day \\
\hline$c_{3}$ & $0.13(\mathrm{~L} / \mathrm{IU})^{\beta} /$ day \\
\hline$c_{4}$ & $0.027 \mathrm{~L} /(\mathrm{IU}$ day $)$ \\
\hline$c_{5}$ & $0.51(\mathrm{~L} / \mathrm{IU})^{\gamma} /$ day \\
\hline$d_{1}$ & 0.50 day $^{-1}$ \\
\hline$d_{2}$ & 0.56 day $^{-1}$ \\
\hline$k_{1}$ & 0.55 day $^{-1}$ \\
\hline$k_{2}$ & 0.69 day $^{-1}$ \\
\hline$k_{3}$ & 0.85 day $^{-1}$ \\
\hline$k_{4}$ & 0.85 day $^{-1}$ \\
\hline$\alpha$ & 0.79 \\
\hline$\beta$ & 0.16 \\
\hline$\gamma$ & 0.02 \\
\hline
\end{tabular}

\begin{tabular}{|l|l||l|l|}
\hline \multicolumn{4}{|c|}{ Eq. (A) } \\
\hline \hline$e_{0}$ & $30 \mathrm{pg} / \mathrm{mL}$ & $p_{2}$ & $0.048 \mathrm{~kL}^{-1}$ \\
\hline$e_{1}$ & $0.11 \mathrm{~L}^{-1}$ & $h_{0}$ & $0.4 \mathrm{IU} / \mathrm{mL}$ \\
\hline$e_{2}$ & $0.21 \mathrm{~L}^{-1}$ & $h_{1}$ & $0.009 \mathrm{IU} /(\mu \mathrm{g} \mathrm{mL})$ \\
\hline$e_{3}$ & $0.45 \mathrm{~L}^{-1}$ & $h_{2}$ & $0.029 \mathrm{IU} /(\mu \mathrm{g} \mathrm{mL})$ \\
\hline$p_{0}$ & $0 \mathrm{ng} / \mathrm{mL}$ & $h_{3}$ & $0.018 \mathrm{IU} /(\mu \mathrm{g} \mathrm{mL})$ \\
\hline$p_{1}$ & $0.048 \mathrm{~kL}^{-1}$ & & \\
\hline
\end{tabular}




\section{Effect of Inhibin Delay on Model Fit and Uniqueness Intervals.}

System (A) and (S) with the inhibin delay is a better approximation to the 28 day Welt data [27] than the model with no delay [23]. The delay model has an asymptotically stable cycle of period 28 days instead of 26 days for the no-delay model. The $L H$ data indicates a 14 day follicular phase and the position and height of the $L H$ surge for the delay model is consistent with that (see Figure 1). Also, the delay $E_{2}$ follicular and luteal peaks are higher then the no-delay. To understand why the inhibin delay is responsible for these differences we examine hormone profiles and ovarian stages for both models over three carefully chosen consecutive cycles. MATLAB simulations of both models were run with the following initial conditions (rounded to two decimal places) given in the order of the 13 state variables in (S), $\{29.65,6.86,8.47,6.15,3.83,11.51,5.48,19.27,45.64,100.73,125.95,135.84$, 168.71\}. The simulations were aligned so that both delay and no-delay periodic orbits are as close to one another as possible at the beginning of their second cycle, indicated by the vertical line at day 29 in Figures 1,2 and 3 . This was done so that the point of our comparison would be the second cycle in these figures and the preceding cycle would also be plotted because hormone profiles during the luteal phase of the preceding cycle influence behavior in our comparison cycle.

The key feature to observe in Figure 2 is that the no-delay FSH (red curve) is higher than the delay FSH from day 19 until day 34, which includes the first six days of the follicular phase of our comparison cycle. Since FSH stimulates follicular development, the no-delay ovarian stages of the second cycle increase sooner than the delay ovarian stages and the no-delay cycle is advanced ahead of the delay cycle (see Figure 3). Nodelay $F S H$ is higher because delay Inh has a greater inhibitory effect than no-delay Inh on FSH synthesis (see (S3)) during that period. Delay Inh (green curve) is greater than no-delay Inh from day 16 to day 24 where the curves cross and then both curves decrease in parallel until day 34. These Inh curves are so close to one another (see Figure 2) from day 24 to day 34 that the delay of 1.5 days results in delay $\operatorname{Inh}(t-1.5)$ being greater than no-delay $\operatorname{Inh}(t)$ for $24 \leq t \leq 34$. Effectively, from day 17.5 to day 34 the synthesis of delay FSH is suppressed more than the synthesis of no delay FSH. This causes the no-delay follicles to develop sooner then the delay follicles with the consequence via (A1) that no-delay $E_{2}$ rises sooner (see Figure 11). Since $E_{2}$ inhibits $F S H$ release (see (S3-S4)), this earlier rise in $E_{2}$ tends to decrease no-delay $F S H$ sooner than delay $F S H$ with the result that the no-delay follicular stages develop to a lesser extent than the delay stages (Figure 3). Also, because $E_{2}$ promotes $L H$ synthesis, the $L H$ surge is earlier and smaller for the no-delay model (Figure 1). The cumulative effect of these profile differences is a shortening of the no-delay cycle length by 2 days and a reduction in no-delay hormone peaks. 

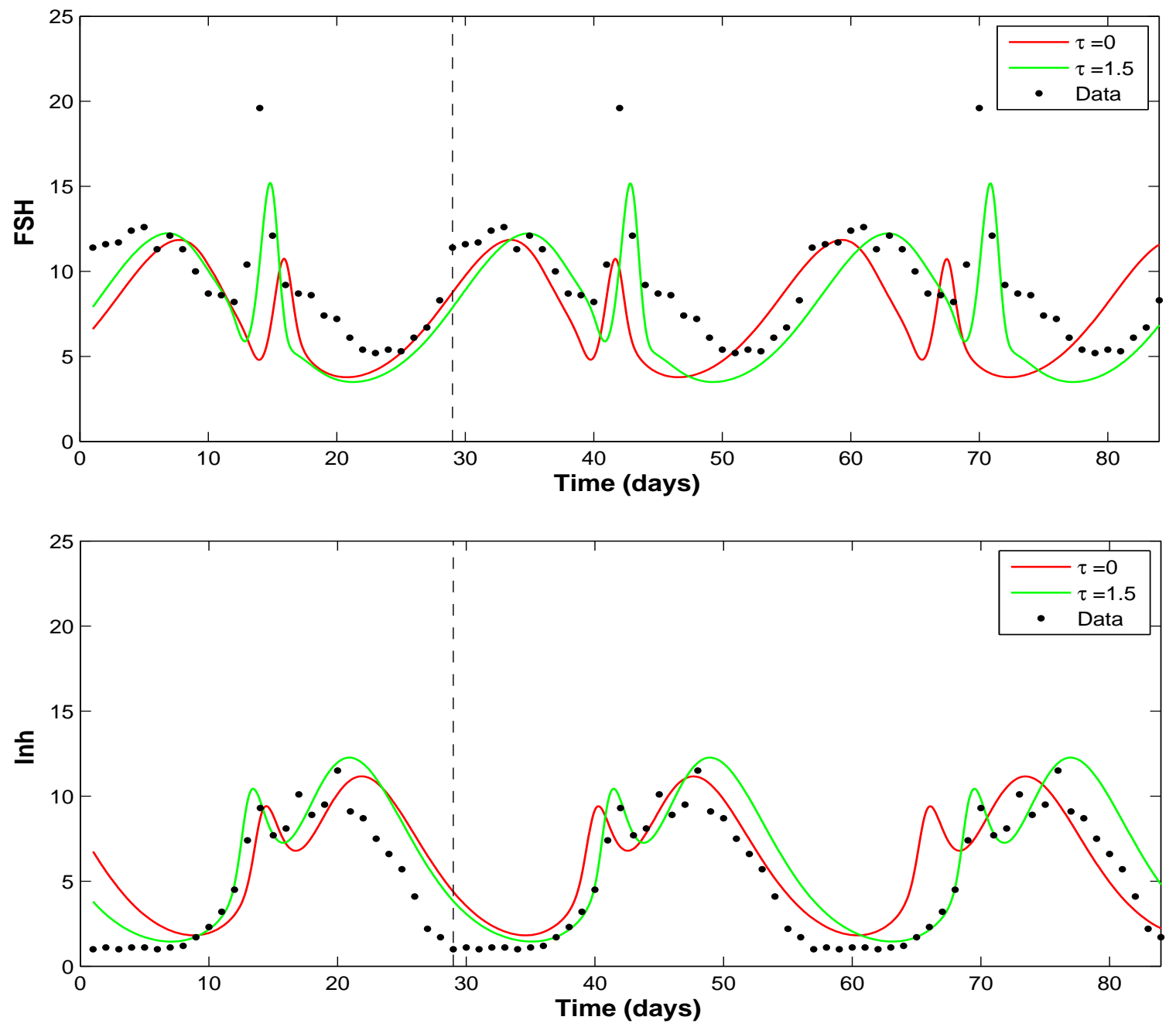

Figure 2: FSH and Inh simulations for 3 consecutive cycles of the delay model (green curves) and the no delay model (red curves) with 84 data points (Welt et al. [27]). The vertical dashed line indicates day 29, the beginning of the second cycle. From day 17.5 to day 34 the synthesis of delay $F S H$ is suppressed more than the synthesis of no delay $F S H$ because of inhibin differences. 

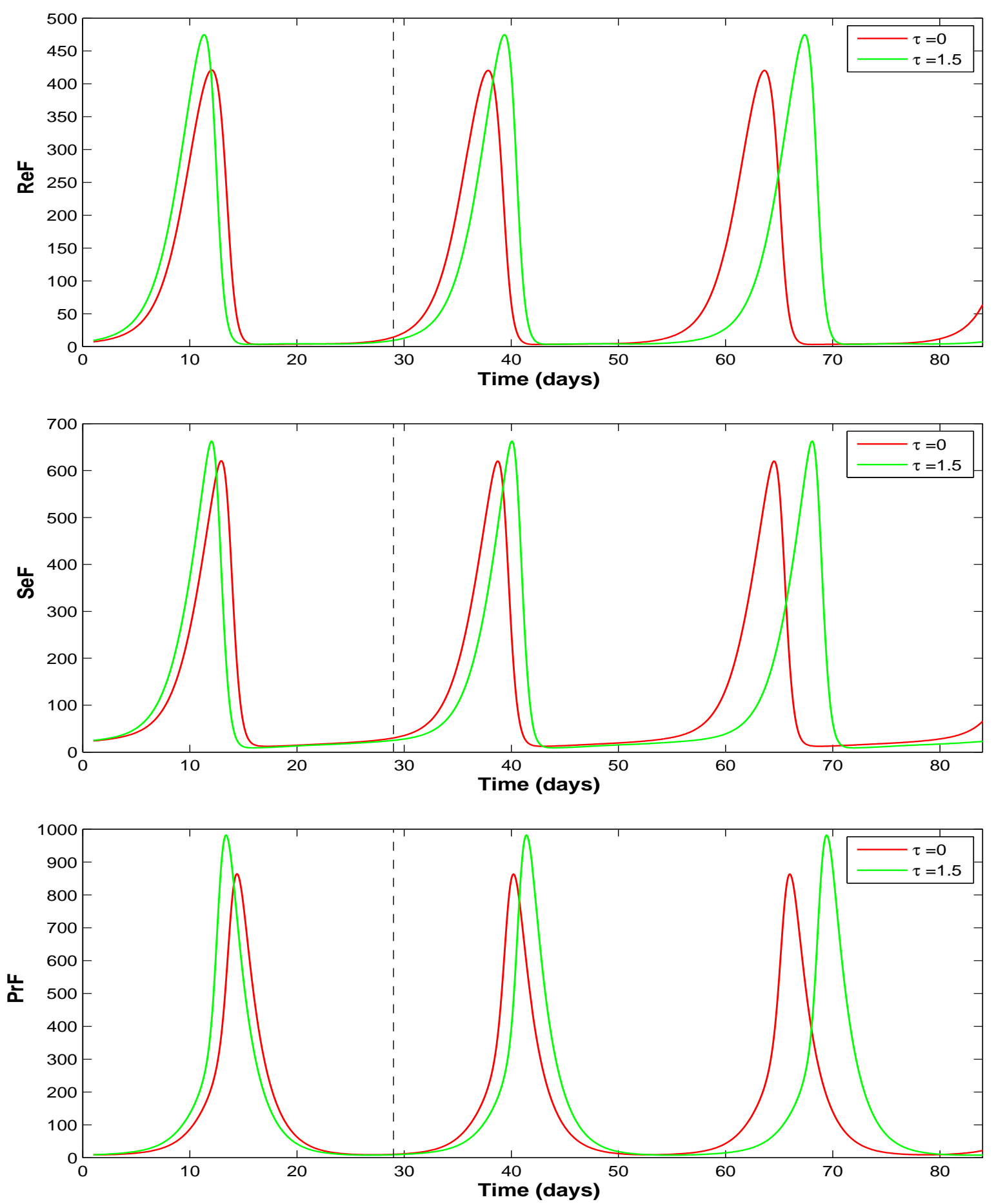

Figure 3: First 3 ovarian stages $R e F, S e F$ and $\operatorname{Pr} F$ for 3 consecutive cycles of the delay model (green curves) and the no delay model (red curves). 
The parameters $K m_{L H}$ and $c_{2}$ are two of the three most sensitive parameters when sensitivity is measured with respect to the $E_{2}$ follicular peak as system output [19, 24. The parameter $K m_{L H}$ is the half-saturation constant in the Hill function in (S1), $\left(V_{1, L H} E_{2}^{8}\right) /\left(K m_{L H}^{8}+E_{2}^{8}\right)$. This sigmoidal shaped function (see Figure 4) acts like a threshold for the synthesis of $L H$ in response to $E_{2}$ blood levels. Once $E_{2}$ concentration reaches the value $K m_{L H}$, half way up the sigmoid as indicated by the dashed line in Figure 4, then the pituitary is synthesizing $L H$ in large amounts, which is necessary for ovulation. For larger values of $K m_{L H}, E_{2}$ must reach a higher level to produce the same $\mathrm{LH}$ synthesis rate. Because higher follicular $E_{2}$ levels may suggest a greater probability of abnormal cycling [23, 24], we construct bifurcation diagrams where $L H$ is plotted against the parameter $K m_{L H}$ to determine the number of stable cycles for a given $K m_{L H}$ value and to determine $L H$ surge height along each cycle. When similar bifurcation diagrams were drawn for the no-delay model [23], an interval of $K m_{L H}$ values was observed for which a unique stable periodic solution existed and it represented an ovulatory cycle. The length of this cycle uniqueness interval varied as the parameter $c_{2}$ was changed [23]. The present study reveals that these uniqueness intervals are larger for the model with inhibin delay, (A) and (S), as indicated in Table 2 . 


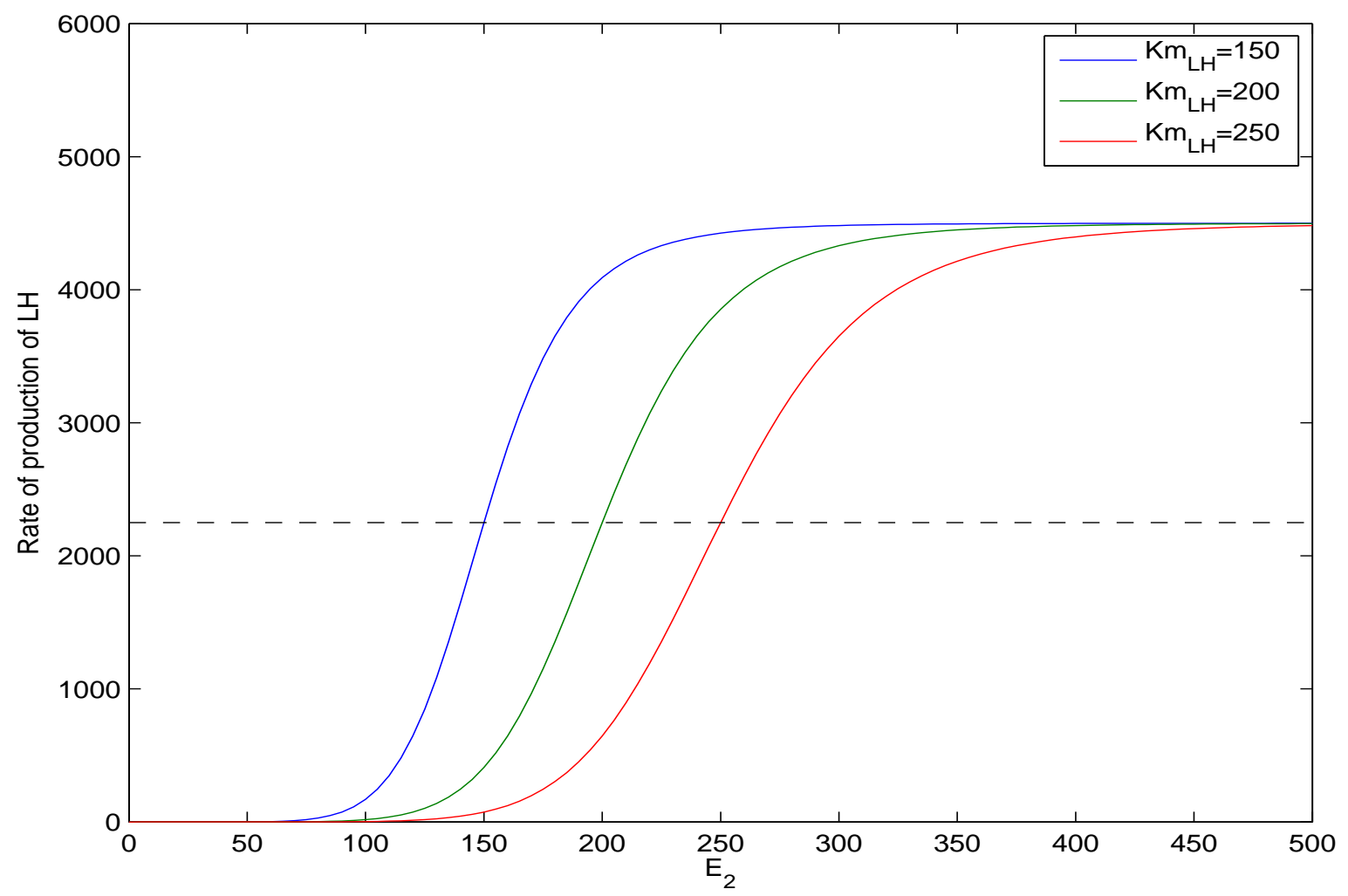

Figure 4: Graphs of Hill functions, $\left(V_{1, L H} E_{2}^{8}\right) /\left(K m_{L H}^{8}+E_{2}^{8}\right)$, for three values of $K m_{L H}$. The dashed line indicates the synthesis rate when $E_{2}=K m_{L H}$, half-saturation. 
Here, the software DDEBIFTOOL [6] is used to construct bifurcation diagrams where the maximal $L H$ value along a periodic solution or at a steady-state solution is plotted against the parameter $K m_{L H}$. Figure 5 displays this bifurcation diagram where the remaining parameters are those in Table 1. Stable and unstable periodic orbits and equilibria are depicted. Saddle-node (SN) and Hopf (HB) bifurcations are labeled. The curve along the lower portion of Figure 5 tracks an equilibrium, which undergoes a destabilizing Hopf bifurcation as $K m_{L H}$ increases through 64 and a stabilizing Hopf bifurcation at $K m_{L H}=248$. The bifurcation at $K m_{L H}=64$ results in a small amplitude, stable, periodic orbit which persists until $K m_{L H}=73$. Stable and unstable cycles appear together at $K m_{L H}=68$ via a saddle-node bifurcation of periodic orbits. The unstable orbit coalesces with the stable Hopf orbit at $K m_{L H}=73$ and both disappear in another saddle-node. The stable cycle appearing at $K m_{L H}=68$ grows in amplitude, continues across the top portion of the diagram and disappears in a saddle-node at $K m_{L H}=282$. This branch of periodic solutions represents the ovulatory cycles of the model (S) with (A), where the $*$ indicates the cycle corresponding to the $K m_{L H}$ value of Table 1, 200 $\mathrm{pg} / \mathrm{ml}$. Analogous behavior occurs at the right side of the bifurcation diagram where the hysteresis character of the curve of periodic orbits is evident. Clearly, for $K m_{L H}$ from 227 to 282 there is a stable, large amplitude ovulatory cycle and a stable, small amplitude non-ovulatory cycle or stable equilibrium. For $K m_{L H}$ in the interval between the lower SN's in Figure 5 ( $\left.73<K m_{L H}<227\right)$, there is only one stable cycle and it is ovulatory. Selgrade [23] referred to this $K m_{L H}$ interval as the cycle uniqueness interval. In the context of this cycle regulation model, a woman's $K m_{L H}$ parameter must fall within her cycle uniqueness interval for her to be assured of only a normal cycle. From Figure 5, we observe that decreasing $K m_{L H}$ from $200 \mathrm{pg} / \mathrm{mL}$ keeps it within the interval and increases the height of the $L H$ surge. However, increasing $K m_{L H}$ to 227 moves $K m_{L H}$ to a region of multiple stable cycles and possible non-ovulation. For $c_{2}=0.07$, the diameter of this cycle uniqueness interval is 154 for the delay model and only 114 for the no-delay model (see Table 2). 


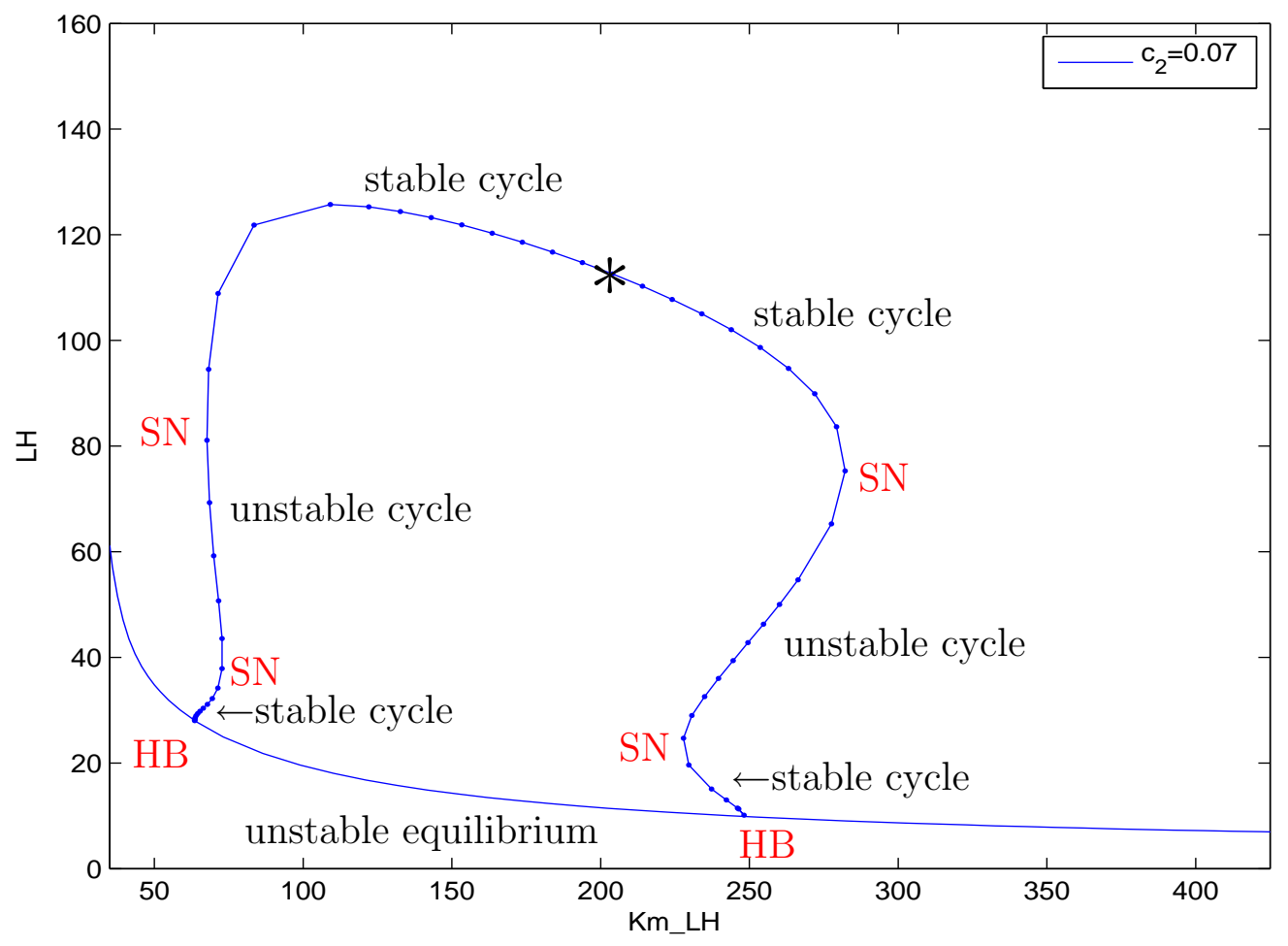

Figure 5: In this bifurcation diagram the maximal $L H$ value along a periodic solution or at an equilibrium is plotted against $K m_{L H}$ when $\tau=1.5$ and $c_{2}=0.07$. HB and SN denote Hopf and saddle-node bifurcations. The $*$ indicates the position of the cycle for the parameters of Table 1 and this cycle is the only stable solution at $K m_{L H}=200 \mathrm{pg} / \mathrm{mL}$. The cycle uniqueness interval is the interval between the lower saddle-nodes, i.e., $73<$ $K m_{L H}<227$. 
Table 2: Size of cycle uniqueness interval for inhibin delay $\tau=0$ (column 2) and $\tau=1.5$ days (column 4) for increasing values of $c_{2} \cdot c_{2}=0.07$ and $K m_{L H}=200 \mathrm{pg} / \mathrm{mL}$ give the best fit to data.

\begin{tabular}{|c|c|c|c|c|}
\hline$c_{2}$ & size $(\tau=0)$ & $K m_{L H}$ bounds $(\tau=0)$ & size $(\tau=1.5)$ & $K m_{L H}$ bounds $(\tau=1.5)$ \\
\hline \hline 0.03 & 126 & $147<K m_{L H}<273$ & 271 & $40<K m_{L H}<311$ \\
\hline 0.04 & 50 & $181<K m_{L H}<231$ & 226 & $44<K m_{L H}<270$ \\
\hline 0.05 & 81 & $153<K m_{L H}<234$ & 173 & $85<K m_{L H}<258$ \\
\hline 0.06 & 118 & $122<K m_{L H}<230$ & 167 & $80<K m_{L H}<247$ \\
\hline 0.07 & 114 & $98<K m_{L H}<212$ & 154 & $73<K m_{L H}<227$ \\
\hline 0.08 & 102 & $84<K m_{L H}<186$ & 141 & $63<K m_{L H}<204$ \\
\hline
\end{tabular}

For the no-delay model, Selgrade [23] investigated how variations in the ovarian transfer parameter $c_{2}$ changed the size of the cycle uniqueness interval. Increasing $c_{2}$ from $c_{2}=0.07$ causes an increased transfer of mass from the first follicular stage $R e F$ to the second stage $S e F$ which diminishes the development of not only ReF but of all subsequent ovarian stages. Effectively, ovarian hormone production is reduced and the cycle uniqueness interval is decreased for both delay and no-delay models. Table 2 lists the cycle uniqueness intervals for various values of $c_{2}$ which we compute for the delay model $(\tau=1.5)$ and which were reported in $\left[23\right.$ for the no-delay model $(\tau=0)$. Decreasing $c_{2}$ from 0.07 in increments of 0.01 widens the cycle uniqueness interval for the delay model but shrinks it for the no-delay model until $c_{2}=0.03$. For the no-delay model as $c_{2}$ decreases, the hysteresis curves enlarge and the Hopf points move closer together resulting in a narrowing of the gap between the lower two saddle-nodes. Then, as described in [23], an unfolding of a transcritical bifurcation of periodic solutions as $c_{2}$ decreases through 0.0305 leads to the disappearance of the left hysteresis curve and a rapid expansion of the cycle uniqueness interval. In contrast, for the delay model, decreasing $c_{2}$ from 0.07 causes the hysteresis curves to enlarge only slightly (see Figure 6), the Hopf points to move apart and the left hysteresis curve to disappear due to two degenerate Hopf bifurcations described below. The uniqueness interval for $\tau=1.5$ when $c_{2}=0.03$ (Figure 7) is over twice as large as that for the no-delay model. 


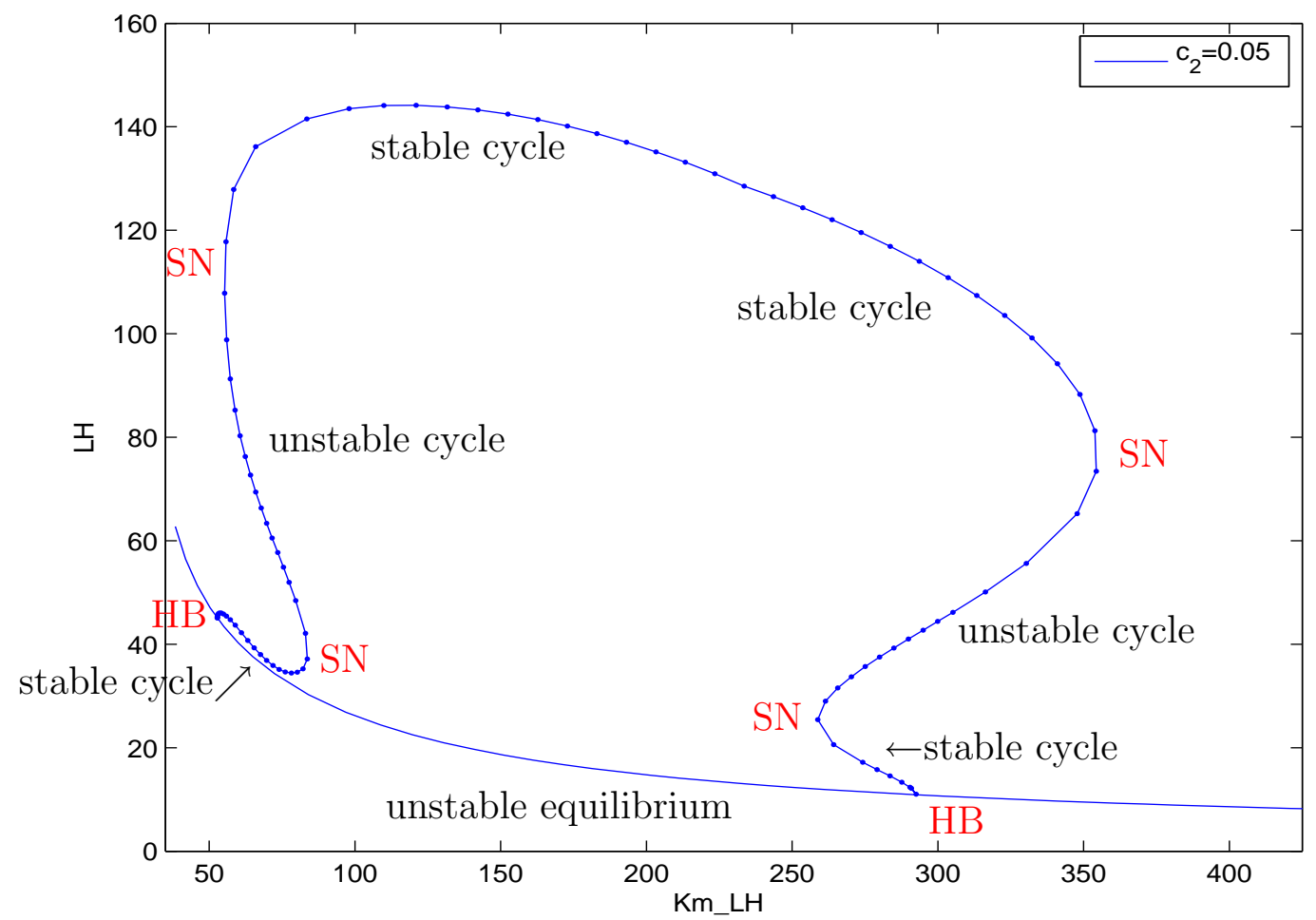

Figure 6: Bifurcation diagram with respect to $K m_{L H}$ when $\tau=1.5$ and $c_{2}=0.05$. HB and SN denote Hopf and saddle-node bifurcations. The length of the cycle uniqueness interval is 173 , i.e., $85<K m_{L H}<258$. 


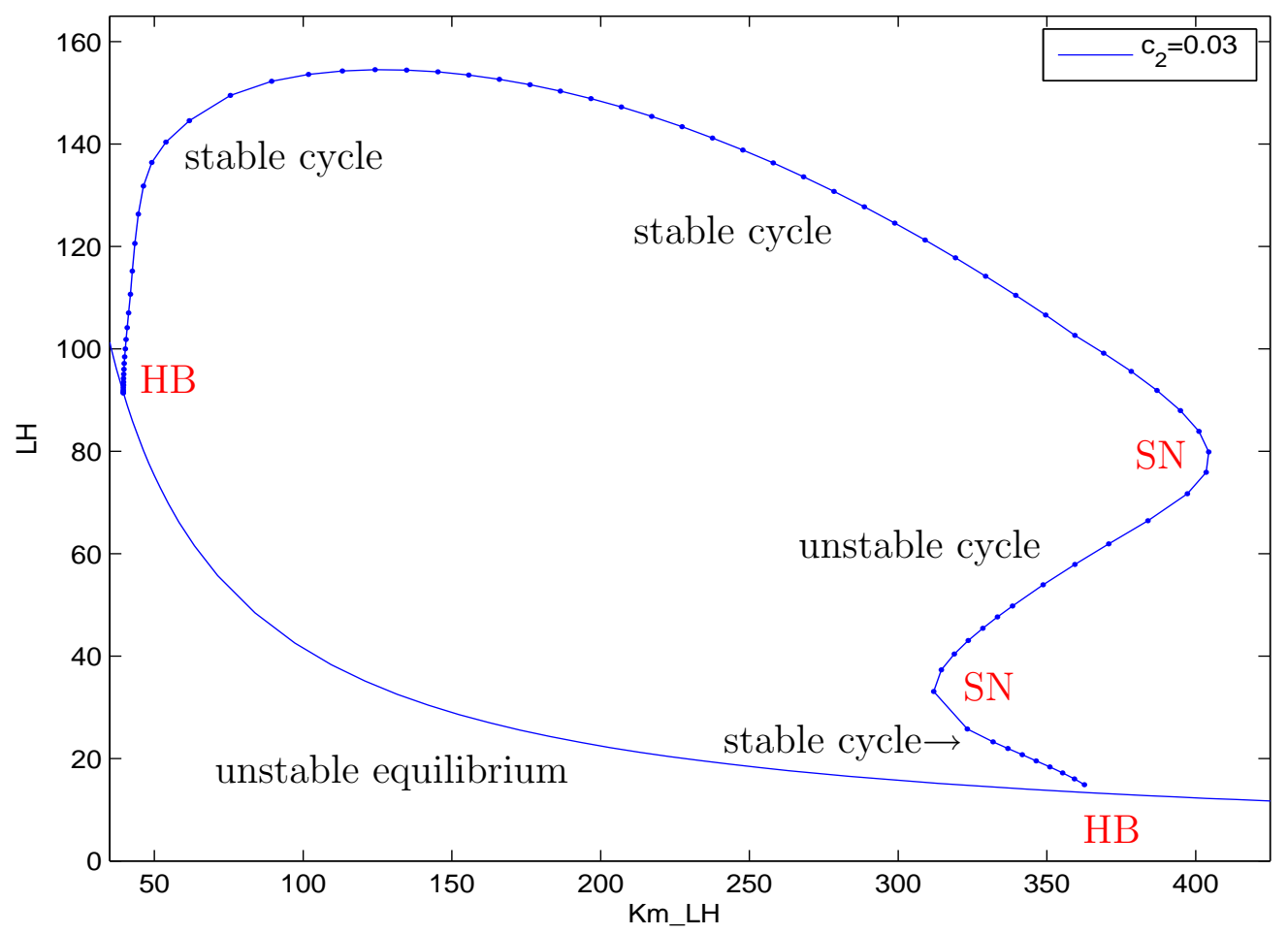

Figure 7: Bifurcation diagram with respect to $K m_{L H}$ when $\tau=1.5$ and $c_{2}=0.03$. $\mathrm{HB}$ and SN denote Hopf and saddle-node bifurcations. The left hysteresis curve has disappeared and the length of the cycle uniqueness interval is 271, i.e., $40<K m_{L H}<311$. 
The broad expansion of the cycle uniqueness interval for $c_{2}$ less than 0.05 is due to two different unfoldings of degenerate Hopf bifurcations which occur for $c_{2}$ near 0.05. Each Hopf bifurcation is degenerate because the real part of the eigenvalue pair crossing the imaginary axis has a zero derivative with respect to the parameter at crossing. One of these degeneracy occurs when two Hopf points coalesce at $c_{2}=0.05147$ and $K m_{L H}=69.8458$. At $c_{2}=0.05$, the left side of Figure 6 displays a branch of stable cycles lying just above a branch of unstable equilibria. Figure 8(a) blows these curves up at $c_{2}=0.051$. They touch when $c_{2}=0.05147$ producing a degenerate Hopf point. Then as $c_{2}$ increases, the degenerate Hopf point separates into two nondegenerate, supercritical Hopf points with stable equilibria in between them pictured at $c_{2}=0.0516$ in Figure 8(b). As discussed in Golubitsky and Schaeffer [8], p. 375, the unfolding of this bifurcation may be described roughly by the equation

$$
-x^{3}+\left(K m_{L H}-69.8458\right)^{2} x+\left(0.05147-c_{2}\right) x=0
$$

where $x$ represents the state variable $L H$ and the line $\{x=0\}$ represents the curve of equilibria. As $c_{2}$ continues to increase above 0.052 , the two Hopf points on the left in Figure 9(a) coalesce in a second degenerate Hopf point at $c_{2}=0.05209$ and $K m_{L H}=$ 61.0174 and that Hopf point disappears for $c_{2}>0.05209$. The unfolding of this bifurcation may be represented by the equation (see [8])

$$
x^{3}+\left(K m_{L H}-61.0174\right)^{2} x+\left(c_{2}-0.05209\right) x=0 .
$$

As $c_{2}$ increases above 0.05209 , the saddle-nodes which determine the cycle uniqueness interval move closer together causing the interval to shrink and move to the left, see Table 2. As $c_{2}$ increases from 0.04 to 0.045 , the left hysteresis curve forms because of the appearance of a kink and two saddle-nodes along the left edge of the large loop of periodic solutions (Figure 10). This kink and the two unfoldings (DegHB1 and DegHB2) are embedded in the continuous display of bifurcation diagrams as $c_{2}$ increases from 0.04 to 0.055 , see Figure 10 . 
(a)

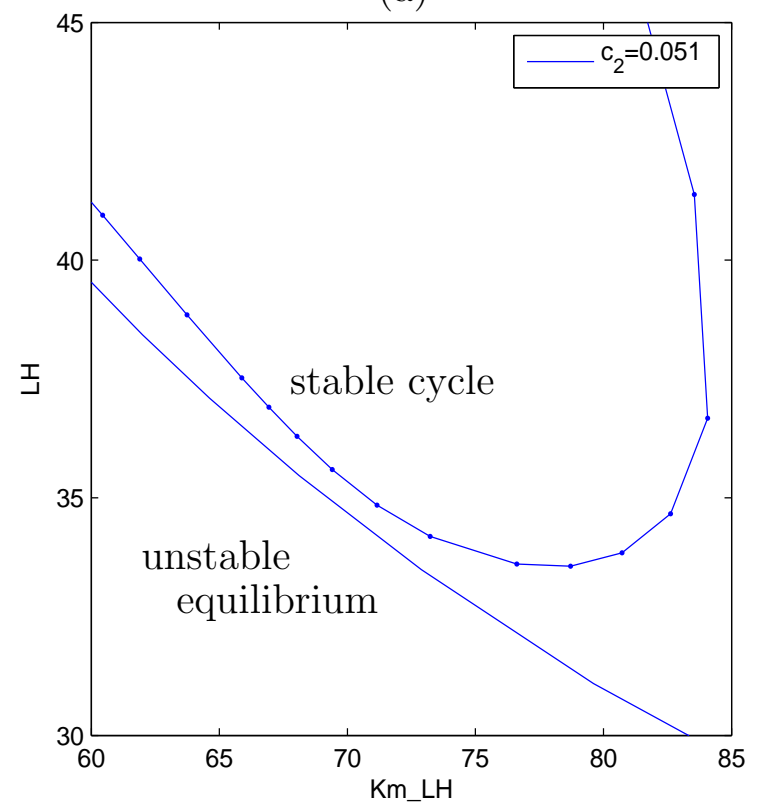

(b)

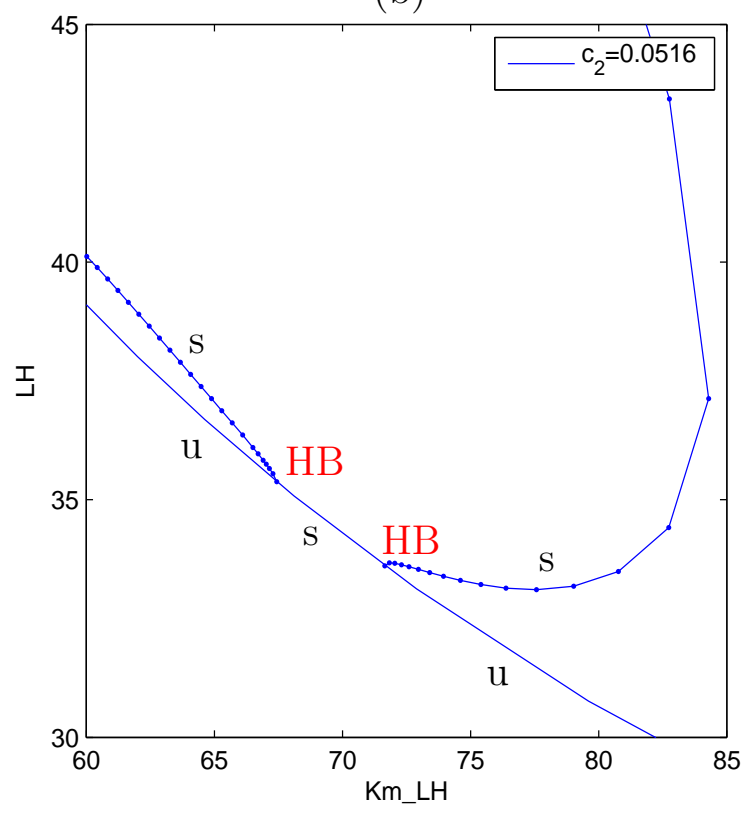

(b): Blow up for $c_{2}=0.0516$

Figure 8: $\quad$ (a): Blow up for $c_{2}=0.051$

HB denotes a Hopf point. 
(a)

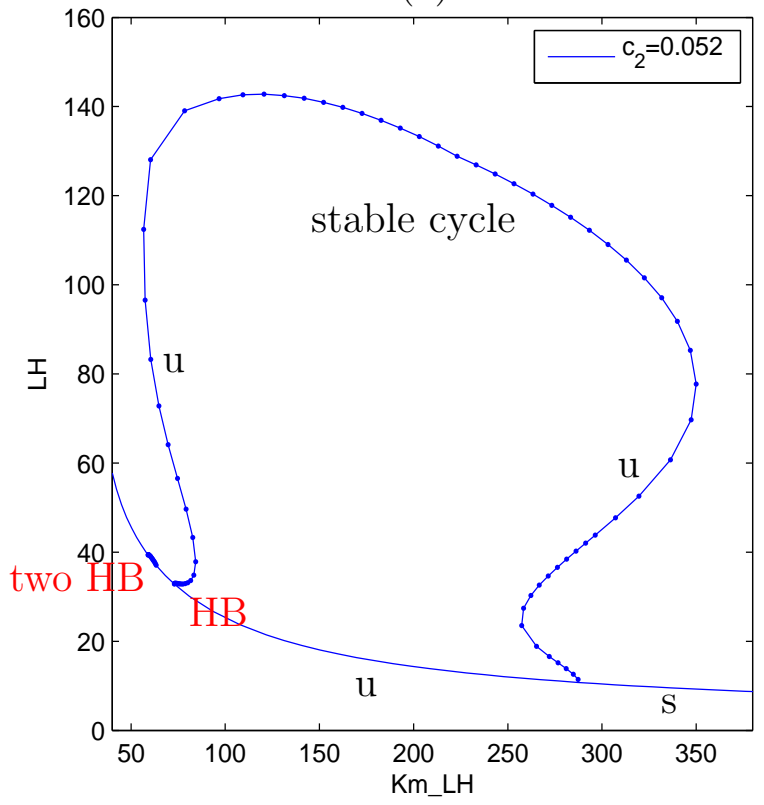

Figure 9: $\quad$ (a): $c_{2}=0.052$ (b)

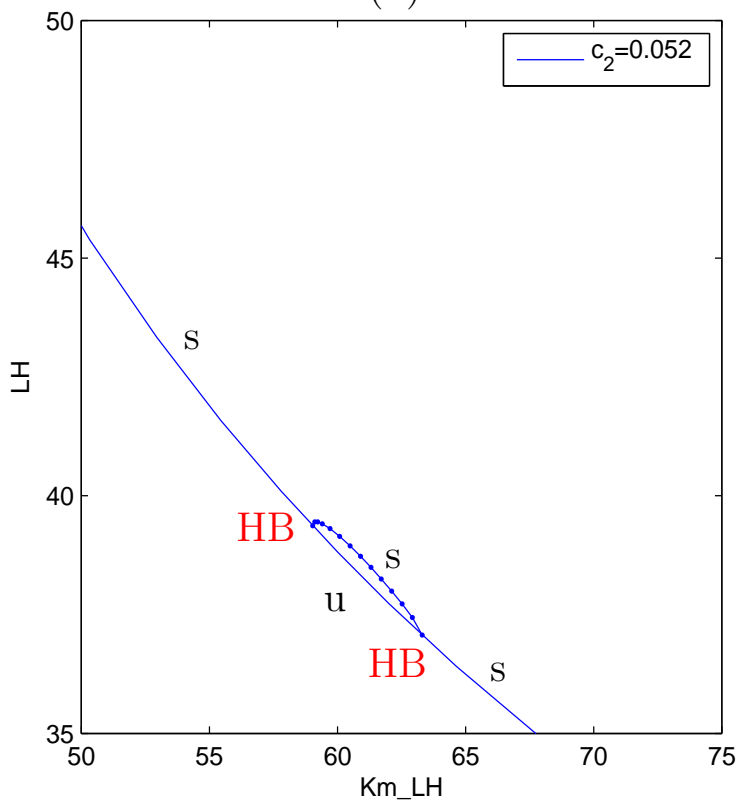
$\mathrm{s}$ indicates stable and $\mathrm{u}$ indicates unstable. HB denotes a Hopf point. 

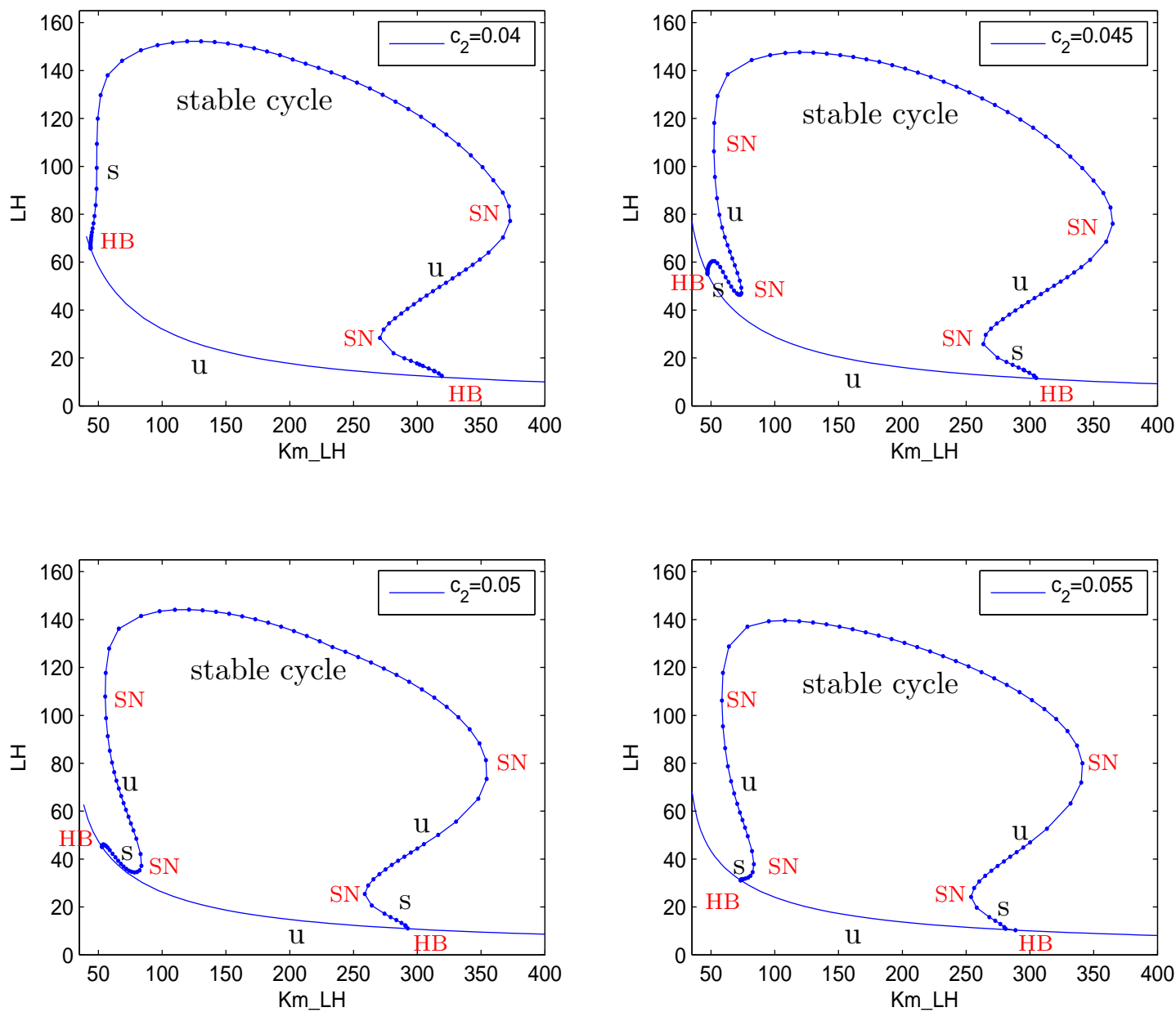

Figure 10: Bifurcation diagrams with respect to $K m_{L H}$ for $\tau=1.5$ as $c_{2}$ increases from 0.04 to 0.055 by increments of 0.5 . A kink appears in the large loop at the left and bends to touch the curve of equilibria causing a degenerate Hopf bifurcation. s indicates stable and $\mathrm{u}$ indicates unstable. [CLICK HERE] for an animated display of bifurcation diagrams as $c_{2}$ increases from 0.04 to 0.055 in increments of 0.001 . 


\section{Comparing Bifurcation Diagrams as Delay $\tau$ Varies.}

For all $c_{2}$ values in Table 2, the delay model has a larger cycle uniqueness interval than the no-delay model. As discussed previously, when $K m_{L H}=200$ and $c_{2}=0.07$ the delay in the effect of inhibin on FSH results in more vigorous growth of ovarian stages, a longer cycle and higher hormone peaks. Numerical simulations indicate that this is also true after reasonable variations in both model parameters, $K m_{L H}$ and $c_{2}$. It is conceivable that the more robust ovarian development of the delay model permits a broader range of half-saturation constants $K m_{L H}$ for the successful surge response of $L H$ to $E_{2}$ priming and, hence, a larger cycle uniqueness interval.

First we fix $c_{2}=0.07$, which is the parameter value fitting the data best (see Table 1). Then we draw bifurcation diagrams with respect to $K m_{L H}$ to study how the cycle uniqueness interval opens up as the delay $\tau$ increase from 0 to 1.5. Figure 11 illustrates these diagrams for $\tau$ values increasing from $\tau=0$ to $\tau=1.5$ by increments of 0.5 . As $\tau$ increases the Hopf points (HB) along the curve of equilibria spread apart as do the saddlenodes (SN), which determine the cycle uniqueness interval. The qualitative features of these diagrams are similar. In particular, there are hysteresis curves on both the left and right edges of a large loop of periodic solutions. The hysteresis curves give rise to two regions of periodic bistability.

For other values of $c_{2}$, these two hysteresis curves do not persist for all values of $\tau$. For instance, if $c_{2}=0.04$ then the hysteresis curve on the left disappears as $\tau$ increases. The cycle uniqueness interval enlarges from 50 when $\tau=0$ to 226 when $\tau=1.5$. The primary reason for this drastic increase is a sequence of bifurcations that occur as $\tau$ increases from 0.7 to 1.2. A degenerate Hopf bifurcation similar to that described by (DegHB2) occurs at $\tau=0.73$ resulting in a bump of stable cycles to the left of the large loop of periodic solutions as pictured in Figure 12. This Hopf bump of stable solutions is just below the branch of unstable cycles in the left hysteresis curve and, as $\tau$ increases, this bump grows and touches the curve of cycles above producing a transcritical bifurcation of periodic solutions in the parameter $K m_{L H}$ when $\tau=1.06$. The unfolding of this transcritical bifurcation is analogous to that discussed in [23] except here the second parameter is $\tau$ instead of $c_{2}$. For $\tau$ values just above 1.06 the bump of stable cycles appears on the other side of the large loop of cycles (see Figure 12) and disappears via the following sequence of bifurcations. At $\tau=1.11$ a degenerate Hopf bifurcation like (DegHB1) causes the Hopf bump to separate from the curve of equilibria producing a small closed loop of periodic solutions (Figure 13). Then this loop shrinks and disappears because the two saddle-nodes at each end of the loop coalesce and annihilate one another at $\tau=1.173$. The unfolding of these bifurcations as $\tau$ increases from 0.7 to 1.2 is animated in Figure 12 . 

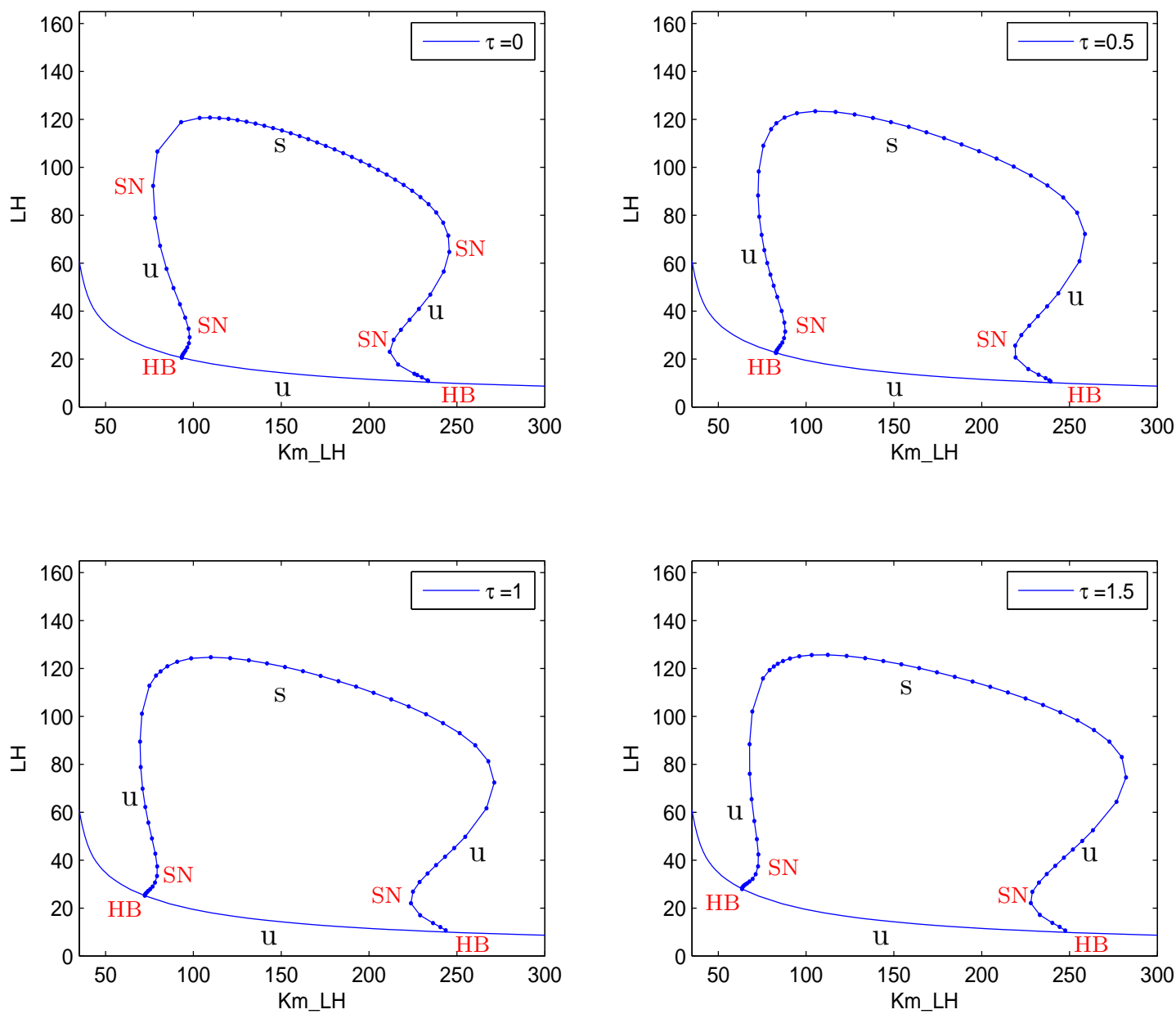

Figure 11: Bifurcation diagrams with respect to $K m_{L H}$ for $c_{2}=0.07$ as $\tau$ increases from $\tau=0$ to $\tau=1.5$ by increments of 0.5 . The cycle uniqueness interval enlarges from 114 to 154. HB and SN denote Hopf and saddle-node bifurcations. s indicates a stable and $\mathrm{u}$, an unstable cycle or equilibrium. 

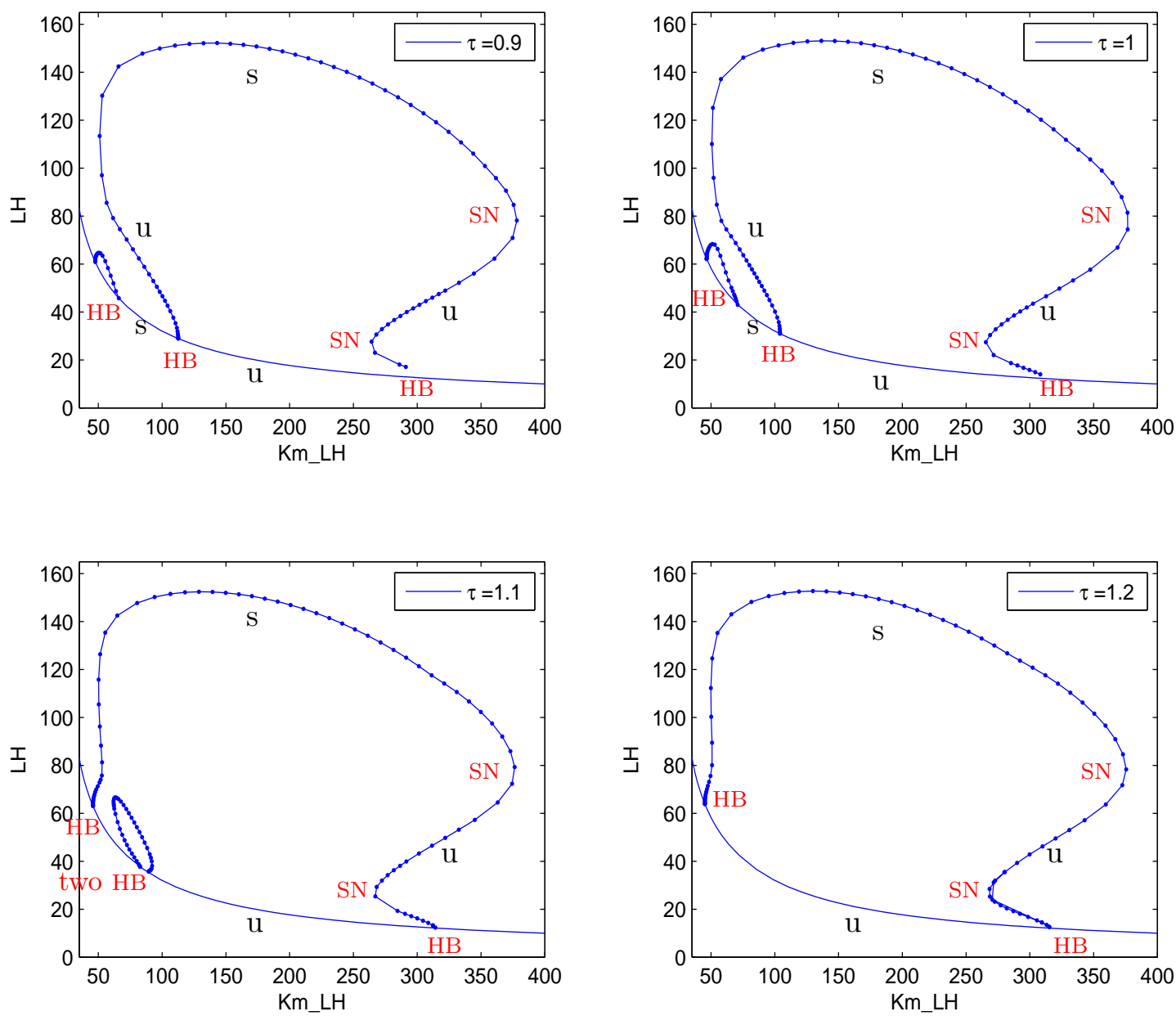

Figure 12: Bifurcation diagrams with respect to $K m_{L H}$ for $c_{2}=0.04$ as $\tau$ increases from $\tau=0.9$ to $\tau=1.2$ by increments of 0.1. HB and SN denote Hopf and saddle-node bifurcations. s indicates a stable and $\mathrm{u}$, an unstable cycle or equilibrium. [CLICK HERE] for an animated display of transcritical and degenerate Hopf bifurcations as $\tau$ increasing from 0.7 to 1.2 in increments of 0.02 . 


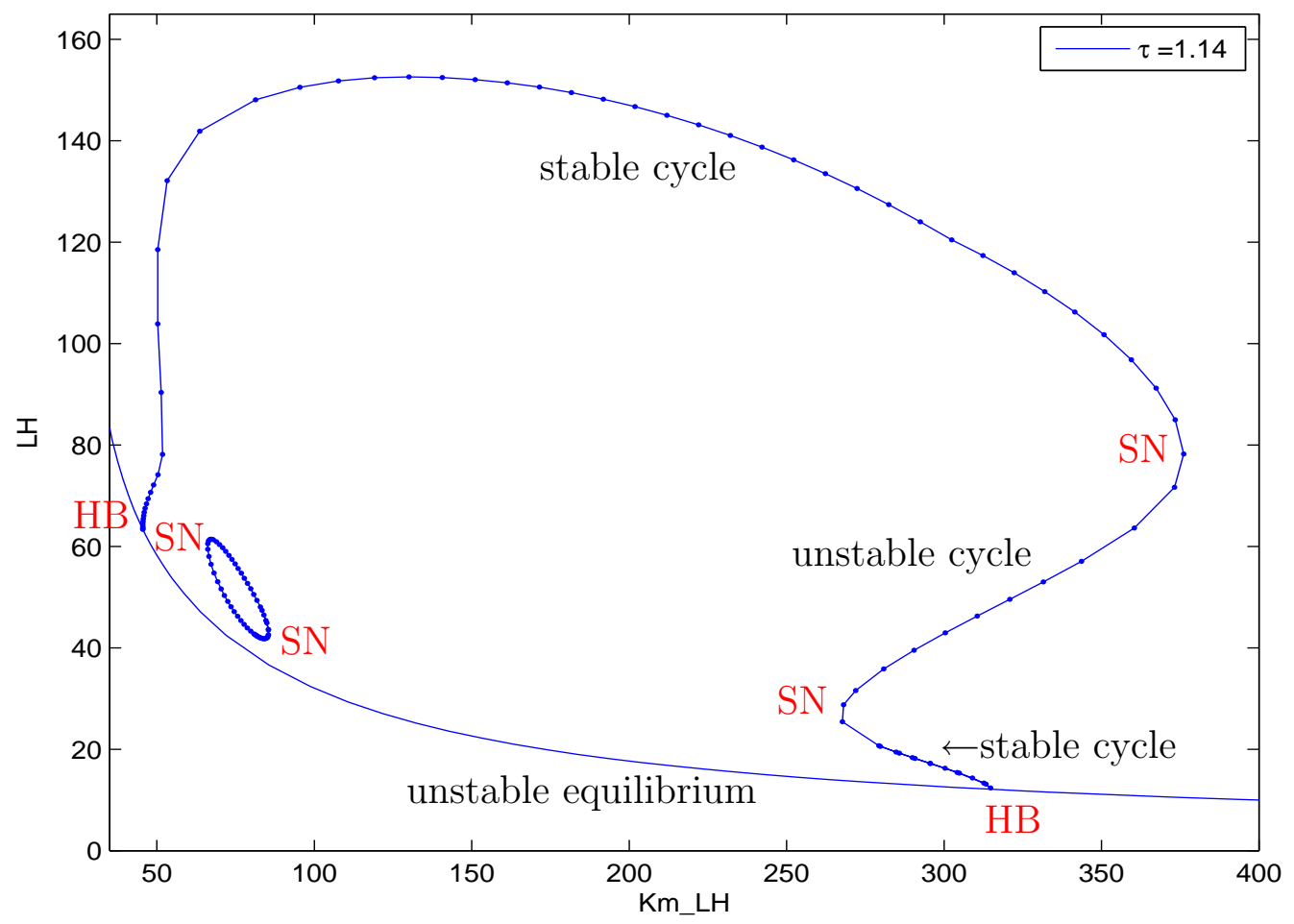

Figure 13: Bifurcation diagram with respect to $K m_{L H}$ when $\tau=1.14$ and $c_{2}=0.04$. HB and SN denote Hopf and saddle-node bifurcations. The small closed loop of cycles has saddle-nodes at each end. 


\section{Summary and Conclusion.}

The half-satuation parameter $K m_{L H}$ in the Hill function in (S1) indicates the level of $E_{2}$ sufficient for significant $L H$ synthesis. We study bifurcation diagrams where maximum $L H$ along a periodic or equilibrium solution is graphed against $K m_{L H}$. We observe an interval of $K m_{L H}$ values for which the model admits a unique stable periodic solution and this solution represents an ovulatory cycle. A large cycle uniqueness interval signifies a wide range of follicular $E_{2}$ levels which promote a $L H$ surge sufficient for ovulation. This cycle uniqueness interval is usually determined by two saddle-nodes bifurcations which lie on hysteresis curves at the left and right sides of the bifurcation diagram.

The parameter $\tau$ is the time delay for the inhibition of FSH synthesis caused by inhibin. We explain why a delay up to 1.5 days (the value of $\tau$ fitting the data best) permits increased ovarian development and a larger interval of $K m_{L H}$ values which result in a unique cycle. The ovarian growth parameter $c_{2}$ promotes mass transfer between the first two stages of ovarian development and is indicative of healthy follicular growth. For various values of $c_{2}$, we illustrate how the cycle uniqueness interval grows as $\tau$ increases due to the occurrences of transcritical and degenerate Hopf bifurcations, e.g., see Figure 12. Also, for delay $\tau$ near 1.5 days, the cycle uniqueness interval increases as $c_{2}$ decreases because of additional growth of the first follicular stage as discussed in Section 3.

The transcritical bifurcation is a prominent feature of the left side of the bifurcation diagrams for smaller values of $\tau$. When this bifurcation is present in the diagram, the cycle uniqueness interval has reduced length, e.g., only 125 for the first frame of Figure 14. The transcritical bifurcation persists for the parameter pairings in Figure 14 until bifurcation point coalesces with a saddle-node for $\tau \approx 1.45$. As $\tau$ increases, the cycle uniqueness interval grows although $c_{2}$ is also increasing (see the animation for Figure 14 ). Hence, a larger delay in the effect of inhibin may compensate for an apparent reduction in growth of the first follicular stage of a cycle. In fact, an increase in FSH inhibition during the luteal phase of the previous cycle due to the delay in inhibin results in greater early follicular development during the next cycle (see the middle cycle in Figure 3). Also, as the delay increases to 1.5 days, the model describes the data in the biological literature [27] better. 

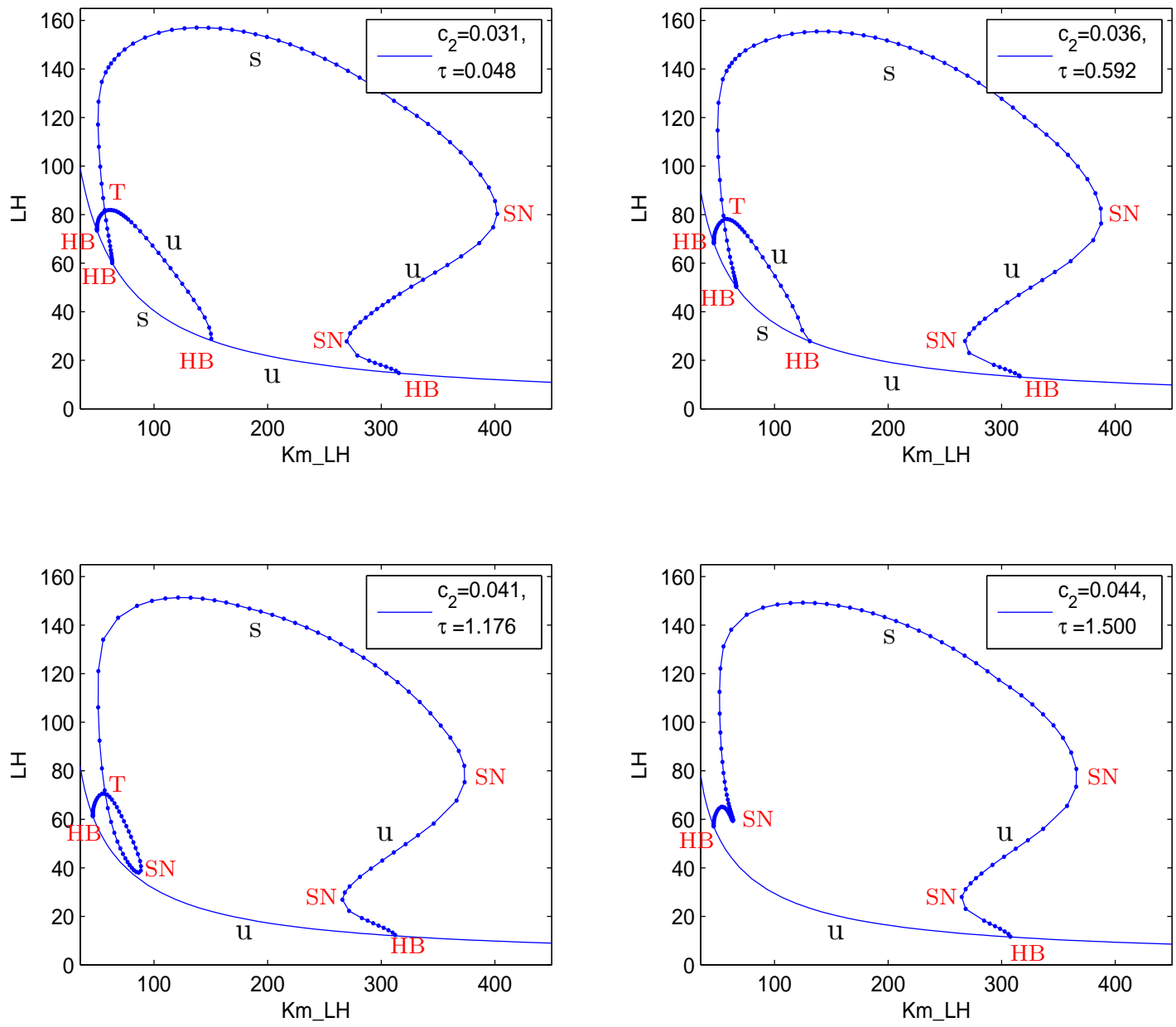

Figure 14: Bifurcation diagrams with respect to $K m_{L H}$ as $\tau$ increases from 0.048 to 1.5 and $c_{2}$ increases from 0.031 to 0.044. HB, SN and T denote Hopf, saddle-node and transcritical bifurcations. $\mathrm{s}$ indicates a stable and $\mathrm{u}$, an unstable cycle or equilibrium. [CLICK HERE] for an animated display of a sequence of transcritical bifurcations revealing the creation of a loop and the broadening of the cycle uniqueness interval as $\tau$ and $c_{2}$ increase. 


\section{References}

[1] F. Alvarez-Blasco, J.I. Botella-Carretero, J.L. San Millan and H.F. Escobar-Morreale (2006). Prevalence and characteristics of the polycystic ovary syndrome in overweight and obese women, Archives of Internal Medicine 166, 2081-2086.

[2] R. Azziz, K.S. Woods, R. Reyna, T.J. Key, E.S. Knochenhauser and B.O. Yildiz (2004). The prevalence and features of the polycystic ovary syndrome in an unselected population, J. Clin. Endocrinol. Metab. 89, 2745-2749.

[3] R.J. Bogumil, M. Ferin, J. Rootenberg, L. Speroff and R.L. Vande Wiele (1972a). Mathematical studies of the human menstrual cycle. I: Formulation of a mathematical model, J. Clin. Endocrinol. Metab. 35, 126-143.

[4] R.J. Bogumil, M. Ferin and R.L. Vande Wiele (1972b). Mathematical studies of the human menstrual cycle. II: Simulation performance of a model of the human menstrual cycle, J. Clin. Endocrinol. Metab. 35, 144-156.

[5] E.J. Doedel (1981). AUTO: A program for the automatic bifurcation analysis of autonomous systems, Congressus Numerantium 30, 265-284.

[6] K. Engelborghs, T. Luzyanina T, and D. Roose (2000). Numerical bifurcation analysis of delay differential equations, Jour. of Comput. and Appl. Math. 125, 265-275.

[7] B. Ermentrout (2002). Simulating, Analyzing, and Animating Dynamical Systems, SIAM, Philadelphia.

[8] M. Golubitsky and D. G. Schaeffer (1985). Singularities and Groups in Bifurcation Theory, Volume 1, Springer-Verlag, New York.

[9] L.A. Harris (2001). Differential equation models for the hormonal regulation of the menstrual cycle, PhD thesis, North Carolina State University, Raleigh, North Carolina. WEB site: www.lib.ncsu.edu/theses/available/etd-04222002$153727 /$ unrestricted/etd.pdf

[10] L. Harris-Clark, P.M. Schlosser and J.F. Selgrade (2003). Multiple stable periodic solutions in a model for hormonal control of the menstrual cycle, Bulletin of Math. Biology 65, 157-173.

[11] J. Hotchkiss and E. Knobil (1994). The menstrual cycle and its neuroendocrine control, The Physiology of Reproduction, Second Edition, E. Knobil and J.D. Neill (Eds), New York: Raven Press, Ltd., pp. 711-750.

[12] F.J. Karsch, D.J. Dierschke, R.F. Weick, T. Yamaji, J. Hotchkiss and E. Knobil (1973). Positive and negative feedback control by estrogen of luteinizing hormone secretion in the rhesus monkey, Endocrinology 92, 799-804.

[13] J. Keener and J. Sneyd (2009). Mathematical Physiology I: Cellular Physiology, Second Edition, New York: Springer-Verlag.

[14] J.H. Liu and S.S.C. Yen (1983). Induction of midcycle gonadotropin surge by ovarian steroids in women: A critical evaluation, J. Clin. Endocrinol. Metab. 57, 797-802. 
[15] J.E.A. McIntosh and R.P. McIntosh (1980). Mathematical Modeling and Computers in Endocrinology, Berlin: Springer-Verlag.

[16] R.I. McLachlan, N.L. Cohen, K.D. Dahl, W.J. Bremner and M.R. Soules (1990). Serum inhibin levels during the periovulatory interval in normal women: Relationships with sex steroid and gonadotrophin levels, Clin. Endocrinol. 32, 39-48.

[17] W.D. Odell (1979). The reproductive system in women, Endocrinology, L.J. DeGroot (Ed), New York: Grune \& Stratton, pp. 1383-1400.

[18] S.R. Ojeda (1992). Female reproductive function, Textbook of Endocrine Physiology, 2nd Ed., J.E. Griffin and S.R. Ojeda (Eds), Oxford: Oxford University Press, pp. $134-188$.

[19] R.D. Pasteur (2008). A multiple-inhibin model for the human menstrual cycle, PhD thesis, North Carolina State University, Raleigh, North Carolina. Web site: http://www.lib.ncsu.edu/theses/available/etd-06102008-194807/

[20] L. Plouffe Jr. and S.N. Luxenberg (1992). Biological modeling on a microcomputer using standard spreadsheet and equation solver programs: The hypothalamic-pituitaryovarian axis as an example, Comput. Biomed. Res. 25, 117-130.

[21] I. Reinecke and P. Deuflhard (2007). A complex mathematical model of the human menstrual cycle, J. Theor. Biol. 247, 303-330.

[22] P.M. Schlosser and J.F. Selgrade (2000). A model of gonadotropin regulation during the menstrual cycle in women: Qualitative features, Environ. Health Perspect. 108(suppl 5), 873-881.

[23] J.F. Selgrade (2010). Bifurcation analysis of a model for hormonal regulation of the menstrual cycle, Math. Biosciences 225, 108-114.

[24] J.F. Selgrade, L.A. Harris and R.D. Pasteur (2009). A model for hormonal control of the menstrual cycle: structural consistency but sensitivity with regard to data, $J$. Theor. Biol. 260, 572-580.

[25] J.F. Selgrade and P.M. Schlosser (1999). A model for the production of ovarian hormones during the menstrual cycle, Fields Institute Communications 21, 429-446.

[26] C.F. Wang, B.L. Lasley, A. Lein and S.S.C. Yen (1976). The functional changes of the pituitary gonadotrophs during the menstrual cycle, J. Clin. Endocrinol. Metab. 42, 718-728.

[27] C.K. Welt, D.J. McNicholl, A.E. Taylor, and J.E. Hall (1999). Female reproductive aging is marked by decreased secretion of dimeric inhibin, J. Clin. Endocrinol. Metab. 84, pp. 105-111.

[28] S.S.C. Yen (1999). Polycystic ovarian syndrome (hyperandrogenic chronic anovulation), Reproductive Endocrinology. Physiology, Pathophysiology and Clinical Management. Fourth Edition, S.S.C. Yen, R.B. Jaffe and R.L. Barbieri (Eds), Philadelphia: W.B. Saunders Co., pp. 436-478. 
[29] S.S.C. Yen (1999). The human menstrual cycle: Neuroendocrine regulation, Reproductive Endocrinology. Physiology, Pathophysiology and Clinical Management. Fourth Edition, S.S.C. Yen, R.B. Jaffe and R.L. Barbieri (Eds), Philadelphia: W.B. Saunders Co., pp. 191-217.

[30] A.J. Zeleznik and D.F. Benyo (1994). Control of follicular development, corpus luteum function, and the recognition of pregnancy in higher primates, The Physiology of Reproduction, Second Edition, E. Knobil and J.D. Neill (Eds), New York: Raven Press, Ltd., pp. 751-782. 